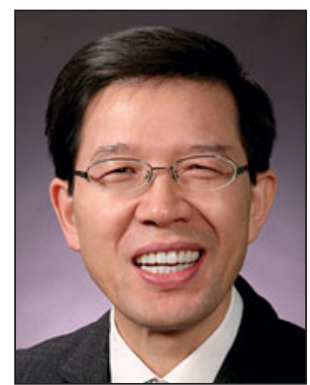

Ce-Wen Nan

Guest Editor for this issue of MRS Bulletin

State Key Lab of New Ceramics and Fine

Processing, and School of Materials

Science and Engineering, Tsinghua University,

China; tel. 86-10-62773587; and

email cwnan@tsinghua.edu.cn.

Nan has been a professor in the School of Materials Science and Engineering at Tsinghua University since 1999. Before joining the faculty of Tsinghua University, he had worked in Wuhan University of Technology, China, since 1985. His recent research focuses on multiferroic materials, thermoelectric oxides, polymer-based composites, and solid-state electrolytes. $\mathrm{He}$ is the Immediate Past President of the International Ceramic Federation. He was elected to the Chinese Academy of Sciences in 2011 and is a Fellow of The Third World Academy of Sciences.

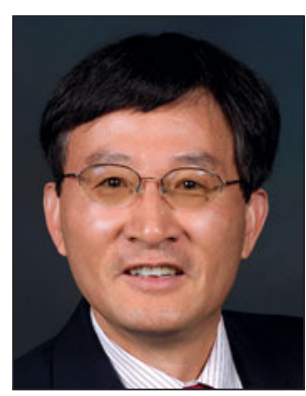

Quanxi Jia

Guest Editor for this issue of MRS Bulletin

Center for Integrated Nanotechnologies,

Los Alamos National Laboratory, USA;

tel. 505-667-2716; and email qxjia@lanl.gov.

Jia is the co-director of the Center for Integrated Nanotechnologies, a Department of Energy Nanoscale Science Research Center operated jointly by Sandia and Los Alamos National Laboratories. He received his BS and MS degrees in electrical engineering from Xian Jiaotong University, China, and his $\mathrm{PhD}$ degree in the same field from The State University of New York (SUNY) at Buffalo. He has authored/co-authored over 400 publications and was awarded 47 US patents. He is a Fellow of the Los Alamos National Laboratory, American Physical Society, Materials Research Society, American Ceramic Society, and American Association for the Advancement of Science.

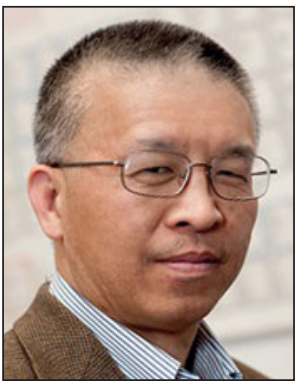

\section{Gang Chen}

Department of Mechanical Engineering,

Massachusetts Institute of Technology, USA;

tel. 617-253-3523; and email gchen2@mit.edu. Chen is the head of the Department of Mechanical Engineering and Carl Richard Soderberg Professor of Power Engineering at Massachusetts Institute of Technology (MIT). He is also the director of MIT's Solid State Solar Thermal Energy Conversion Center ( ${ }^{3} \mathrm{TEC}$ ), an Energy Frontier Research Center funded by the US Department of Energy. He received an NSF Young Investigator Award, an R\&D 100 Award, a Heat Transfer Memorial Award, and a Nukiyama Memorial Award. He is a Fellow of American Association for the Advancement of Science, American Physical Society, American Society of Mechanical Engineers, and the Guggenheim Foundation. $\mathrm{He}$ is an academician of Academia Sinica and a member of the US National Academy of Engineering.

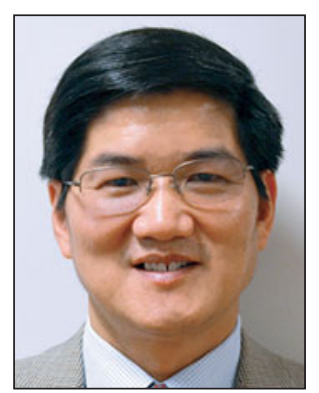

\section{Long-Qing Chen}

The Pennsylvania State University, USA

tel. 814-863-8101; and email lqc3@psu.edu. Chen is the Donald W. Hamer Professor of Materials Science and Engineering, professor of engineering science and mechanics, and professor of mathematics at The Pennsylvania State University (Penn State). He received his BS degree from Zhejiang University, China; MS degree from SUNY at Stony Brook; and PhD degree from the Massachusetts Institute of Technology, all in materials science and engineering. His main research interests are in the area of computational microstructure evolution and multiscale modeling of metallic alloys, oxide thin films and multiferroic heterostructures, and energy materials. He is a Fellow of the Materials Research Society (MRS), American Physical Society, and American Society for Metals. He was awarded a University Faculty Scholar Medal and Distinguished Professorship at Penn State, a Guggenheim Fellowship, and an ASM Materials Research Silver Medal. He received the 2011 TMS EMPMD Distinguished Scientist Award and the 2014 MRS Materials Theory Award.

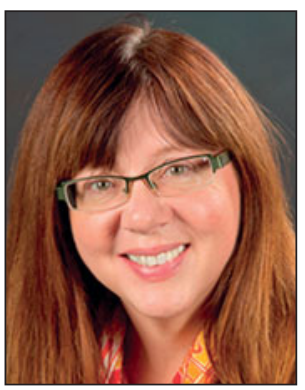

\section{Millicent Anne Firestone}

Materials Physics \& Applications Division,

Center for Integrated Nanotechnologies,

Los Alamos National Laboratory, USA;

tel. 505-665-8554; and email firestone@lanl.gov. Firestone is the thrust leader for soft, biological, and composite nanomaterials at the Center for Integrated Nanotechnologies, Los Alamos National Laboratory (LANL). Prior to joining LANL in 2013, she led the nanostructured biocomposite materials program in the Materials Science Division at Argonne National Laboratory from 2002 to 2013. She received her $\mathrm{PhD}$ degree in chemistry from Northwestern University in 1993. Her research centers on developing singlestep syntheses for the production of nanoparticle-polymer composites and studying composite structure with synchrotron $x$-ray scattering.

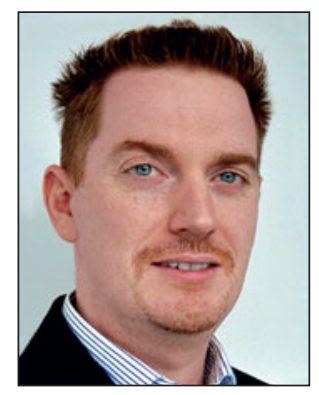

\section{Stephan Götzinger}

Department of Physics, Friedrich-Alexander University Erlangen-Nuremberg; and Max Planck Institute for the Science of Light, Germany; email stephan.goetzinger@mpl.mpg.de. Götzinger has been an associate professor in the Department of Physics at Friedrich-Alexander University Erlangen-Nuremberg since 2012. He studied physics and mathematics at the University of Kaiserslautern, Germany. In 2004, he earned his $\mathrm{PhD}$ degree in physics from the Humboldt University of Berlin. After a postdoctoral stay at Stanford University, he became a senior scientist at ETH Zurich, Switzerland, where he received the Venia Legendi in the Department of Chemistry and Applied Biosciences in 2011. His research is focused on solid-state quantum optics. Current research interests include highly efficient single-photon sources, efficient coupling of single photons to single emitters, cavity quantum electrodynamics, and plasmonics.

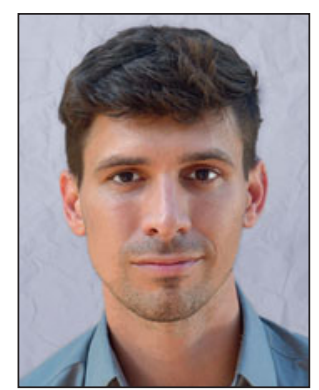

Steven C. Hayden

Materials Physics \& Applications Division, Center for Integrated Nanotechnologies, Los Alamos National Laboratory, USA; email scchayden@gmail.com.

Hayden is a postdoctoral researcher at Los Alamos National Laboratory. He received his $\mathrm{PhD}$ degree in chemistry (nanomaterials) from Georgia Institute of Technology in 2012, where he investigated the use of plasmonic nanoparticles in biological and biomimetic systems for applications in solar energy conversion, imaging, and therapeutics. His research interests focus on self-assembled, bioinspired systems with mesostructured architectures as a means to arrange both synthetic and natural nanoscale species in order to generate responsive composites with emergent, tunable optical, and electronic properties. 


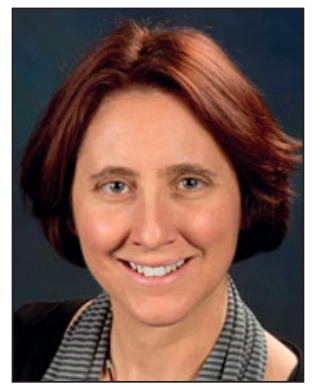

Jennifer A. Hollingsworth

Center for Integrated Nanotechnologies,

Los Alamos National Laboratory, USA

tel. 505-665-0399; and email jenn@lanl.gov.

Hollingsworth is a scientist with the Center for Integrated Nanotechnologies, Los Alamos National Laboratory (LANL). She obtained her BA degree in chemistry from Grinnell College in 1992 and a PhD degree in materials inorganic chemistry from Washington University in St. Louis in 1999. She joined LANL as a Director's Postdoctoral Fellow in 1999, becoming a staff scientist in 2001 and winning a Distinguished Postdoctoral Performance Award (Small Team) in 2002. In 2013, she was awarded a LANL Fellows' Prize for Research for her discovery and elaboration of non-blinking "giant" quantum dots. She is engaged in improving the methods for complex hetero-nanomaterials synthesis through new approaches to automation and novel growth mechanisms, and fostering collaborations with theorists and experimentalists toward establishing predictive capabilities for nanomaterials development.

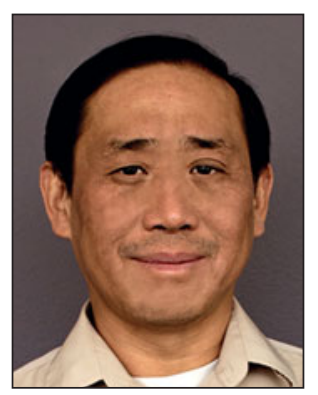

Han Htoon

Center for Integrated Nanotechnologies,

Los Alamos National Laboratory, USA;

tel. 505-665-5444; and email htoon@lanl.gov.

Htoon is a scientist in the Center for Integrated Nanotechnologies at the Los Alamos National Laboratory (LANL). He received his BS and MS degrees in physics from the University of Yangon, Myanmar, and Western Illinois University, respectively. He earned his $\mathrm{PhD}$ degree in physics from The University of Texas at Austin in 2001. He joined LANL as a Director's Postdoctoral Fellow in 2001, and became a staff scientist in 2005 after winning a Distinguished Postdoctoral Performance Award. His research interests include fundamental photophysics and quantum optics of semiconductor nanostructures, photonic and plasmonic, development of nanoscale optical spectroscopy approaches, and integration of nanomaterials toward functional structures and prototype devices.

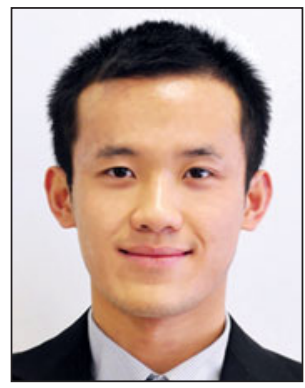

Jia-Mian Hu

The Pennsylvania State University, USA; tel. 814-865-0389; and email juh34@psu.edu. $\mathrm{Hu}$ is a postdoctoral associate in the group of Long-Qing Chen at The Pennsylvania State University. He received his BS degree in materials physics from Sichuan University, China, in 2008, and his PhD degree in materials science and engineering from Tsinghua University, China, in 2013 under the supervision of Ce-Wen Nan. His research interests focus on multiferroic magnetoelectric heterostructures, with a particular interest in the application of phase-field models to magnetoelectric device designs. He is the recipient of a 2011 Materials Research Society Graduate Student Gold Award and the 2012 Graduate Excellence in Materials Science Diamond Award.

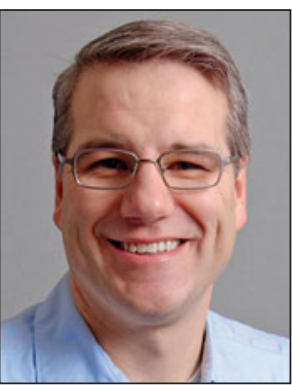

\section{Dale L. Huber}

Center for Integrated Nanotechnologies,

Sandia National Laboratories, USA;

email dale.huber@sandia.gov.

Huber is a principal member of the technical staff at the Center for Integrated Nanotechnologies at Sandia National Laboratories. He received his $\mathrm{PhD}$ degree in polymer science from the University of Connecticut in 2000. His research explores the solution synthesis of a wide range of both organic and inorganic materials, including nanoparticle synthesis via controlled nucleation and growth, in situ polymerization of monolayers, and films on particles and microsystem surfaces.

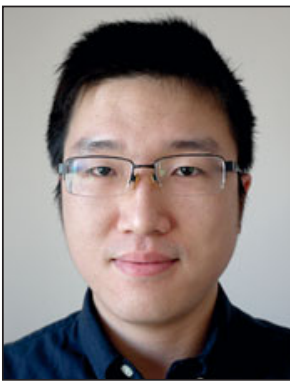

Bolin Liao

Department of Mechanical Engineering,

Massachusetts Institute of Technology, USA; tel.617-324-2068; and email bolin@mit.edu. Liao is a PhD candidate in the Department of Mechanical Engineering at Massachusetts Institute of Technology (MIT) under the supervision of Gang Chen. He graduated from Tsinghua University, China, in 2010, with a bachelor of engineering degree in microelectronics, and obtained his master's degree in mechanical engineering from MIT in 2012. His main research interests are nanoscale transport phenonema of electrons, phonons, and magnons, and their applications in solid-state energy systems. He has served as a referee for journals including Physical Review Letters, Energy \& Environmental Science, and Physical Review B: Condensed Matter.

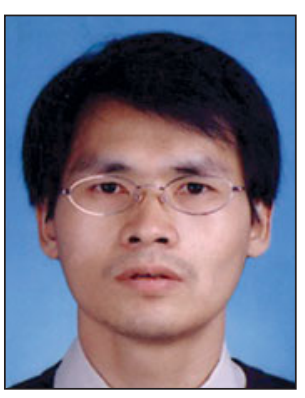

\section{Yuanhua Lin}

State Key Lab of New Ceramics and Fine Processing, School of Materials Science and Engineering, Tsinghua University, China; email linyh@tsinghua.edu.cn.

Lin is the Changjiang Scholar Distinguished Professor of Materials Science at the School of Materials Science and Engineering, Tsinghua University. He received his BS degree from the East China Institute of Technology, his MS degree from the Institute of Process and Engineering Chinese Academy of Sciences, and his PhD degree from Tsinghua University. He was a scholar for the Japan Society for the Promotion of Science at the University of Tokyo in 2005. His main research interests include high-к ceramics and thin films for high energy density capacitors applications; oxide-based diluted magnetic semiconductor thin films; and high-temperature oxide thermoelectric materials and devices for energy conversion. He has over 200 publications and 10 patents in these areas.

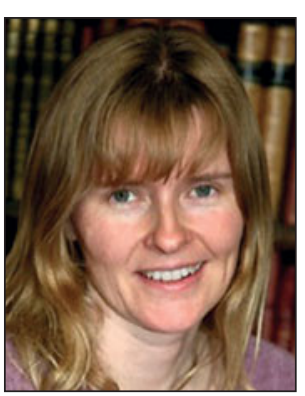

\section{Judith L. MacManus-Driscoll}

Department of Materials Science and Metallurgy, University of Cambridge, UK; tel. +44 (0)1223-334468; and

email jld35@cam.ac.uk.

Driscoll is a professor in materials science at the University of Cambridge and is a long-term visiting staff member at Los Alamos National Laboratory. Her research focuses on oxide thin films for electronics and energy. She is a Fellow of the Institute of Physics, the American Physical Society, and the Materials Research Society. She is the founding editor of the journal $A P L$ Materials from the American Institute of Physics. She has over 300 papers and also a number of patents that have been licensed by industry.

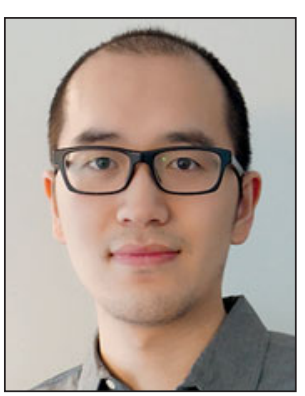

Tianxiang Nan

Northeastern University, USA;

tel. 617-515-6065; and

email nan.t@husky.neu.edu.

Nan is a PhD candidate in Nian X. Sun's laboratory at Northeastern University. His research interests focus on magnetic materials, spintronics, magnetoelectric heterostructures, and its application on microelectromechanical system resonant magnetometers. He is the recipient of the 2014 Chinese Government Award for Outstanding Students Abroad, and was nominated for the Best Presentation Awards at the IEEE Intermag 2013 and MMM 2013 conferences. 


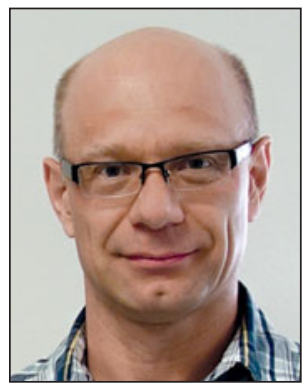

\section{Andrei Piryatinski}

Los Alamos National Laboratory, USA

tel. 505-665-3639; and email apiryat@lanl.gov.

Piryatinski is a technical staff member at Los

Alamos National Laboratory (LANL). He earned his $\mathrm{PhD}$ degree in physics and optical sciences from the University of Toledo in 1997. He performed his postdoctoral training at the University of Rochester from 1997 to 1999, and at the University of Wisconsin-Madison from 1999 to 2002. In 2002, he joined LANL as a CNLS postdoc prior to becoming a technical staff member in 2006. His research covers theoretical study of ultrafast carrier and phonon dynamics in nanostructured materials, their optical and transport properties, and their ultrafast nonlinear spectroscopy.

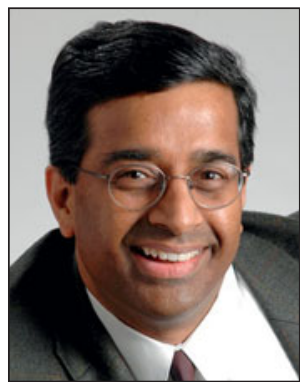

Ramamoorthy Ramesh

Department of Materials Science and

Engineering and Department of Physics, University of California, Berkeley, USA; and Lawrence Berkeley National

Laboratory, USA; tel. 510-642-2347; and email rramesh@berkeley.edu.

Ramesh is the associate lab director at Lawrence Berkeley National Laboratory and the Purnendu Chatterjee Chair Professor of Physics and Materials Science at the University of California, Berkeley. He received his $\mathrm{PhD}$ degree from the University of California, Berkeley in 1987. From 1995 to 2004, he was a distinguished university professor at the University of Maryland College Park. His awards include the Materials Research Society David Turnbull Lectureship Award in 2007 and the 2010 APS McGroddy New Materials Prize. In 2009, he was elected Fellow of MRS, and in 2011, he was elected to the National Academy of Engineering.

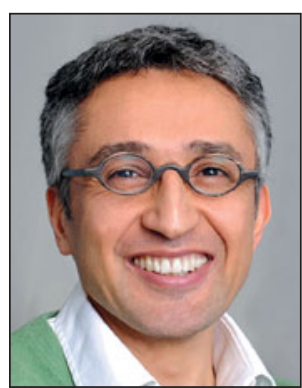

\section{Vahid Sandoghdar}

Max Planck Institute for the Science of Light; and Friedrich-Alexander University ErlangenNuremberg, Germany; tel. +49 91316877 200; and email vahid.sandoghdar@mpl.mpg.de. Sandoghdar obtained his BS degree in physics from the University of California, Davis in 1987 and a PhD degree in physics from Yale University in 1993. After a postdoctoral stay at the Ecole Normale Supérieure in Paris, he moved to the University of Konstanz in Germany, where he started a new line of research to combine single molecule spectroscopy and scanning probe microscopy. In 2001, he accepted a chair at the Laboratory of Physical Chemistry at ETH Zurich, Switzerland. In 2011, he became director of the Max Planck Institute for the Science of Light and Alexander von Humboldt Professor at the University of Erlangen-Nuremberg in Germany. His current research ranges from quantum optics, plasmonics, and ultrahigh resolution microscopy, to nanobiophysics.

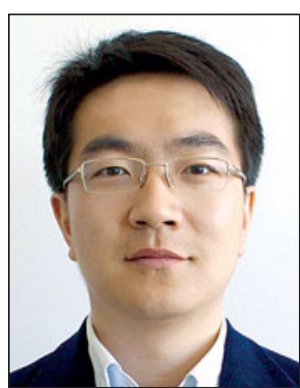

\section{Yang Shen}

School of Materials Science and

Engineering, Tsinghua University,

China; tel. 86-10-62794855; and

email shyang_mse@tsinghua.edu.cn.

Shen is an associate professor of materials science and engineering at Tsinghua University. Before joining the faculty of Tsinghua University in 2011, he worked as a postdoctoral researcher at the University of California, Santa Barbara; and Harvard University. For the past 10 years, he has been focusing on the fundamentals and applications of polymer nanocomposites dielectrics and related energy storage devices. He has published over 70 papers and holds five patents in this area.

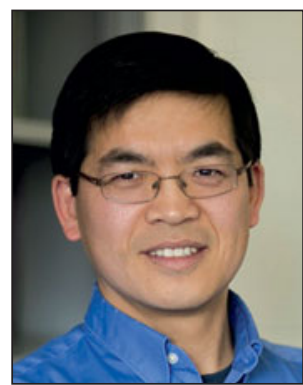

Nian X. Sun

Northeastern University, USA

tel. 617-373-3351; and

emailn.sun@neu.edu.

Sun is a professor in the Electrical and Computer Engineering Department and the director of the W.M. Keck Laboratory for Integrated Ferroics at Northeastern University. His research has been on integrated magnetics and multiferroics for sensing, memory, power, RF, and microwave electronics. He was the recipient of an NSF CAREER Award, ONR Young Investigator Award, Søren Buus Outstanding Research Award, USAF Summer Faculty Fellowship, and the first prize IDEMA Fellowship. He has over 180 publications, and more than 20 patents and patent disclosures. He is an editor of IEEE Transactions on Magnetics and is a Fellow of IOP and IET.

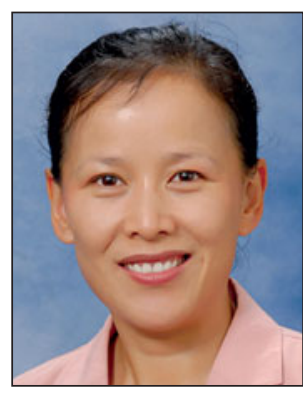

\section{Haiyan Wang}

Department of Electrical and Computer Engineering, Texas A\&M University,

USA; tel. 979-845-5082; and

email wangh@ece.tamu.edu.

Wang is a full professor in the Department of Electrical and Computer Engineering at Texas A\&M University. She received her PhD degree from North Carolina State University in 2002. From 2003 to 2006, she was on the staff of Los Alamos National Laboratory, first as a postdoctoral fellow, and then as a technical staff member. In 2006, she moved to Texas A\&M as an assistant professor and was promoted to associate professor and full professor in 2010 and 2014, respectively. In 2013-2015, she served as a program director at the National Science Foundation, returning to Texas A\&M in 2015. She has received several major awards and recognitions, including ACerS Fellow 2015, ASM Fellow in 2014, TAMEST O'Donnell Award in 2015, Presidential Early Career Award for Scientists and Engineers in 2008, NSF CAREER Award in 2009, Office of Naval Research Young Investigator Program in 2008, and the Air Force Research Laboratory Young Investigator Research Program in 2007.

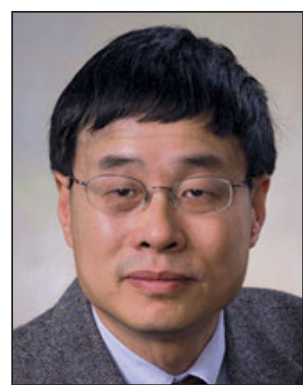

\section{Qiming Zhang}

The Pennsylvania State University,

USA; email gxz1@psu.edu.

Zhang is a distinguished professor of engineering at The Pennsylvania State University (Penn State). His research interests include fundamentals and applications of electronic and electroactive materials. During his more than 20 years at Penn State, he has conducted research covering actuators, sensors, transducers, dielectrics and charge storage devices, polymer thin-film devices, polymer MEMS, electrocaloric-effect and solid-state cooling devices, and electro-optic and photonic devices. He has over 400 publications and 15 patents in these areas. He is a Fellow of IEEE and APS.

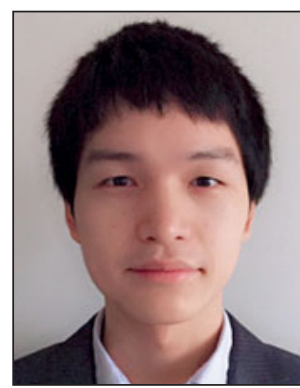

Wenrui Zhang

Department of Materials Science and

Engineering, Texas A\&M University, USA; email zhwrican@tamu.edu.

Zhang is a PhD candidate in the Department of Materials Science and Engineering at Texas A\&M University, supervised by Haiyan Wang. He received his $B S$ degree in materials science from Wuhan University of Science and Technology, China, in 2011. His current research focuses on multifunctional oxide nanocomposite thin films and electronic and magnetic device applications. 